\title{
Finding Light in Dark Atomic Clouds
}

\author{
Researchers have prepared and manipulated subradiant states-in which \\ collective effects slow down the decay of excited atoms-in a dense \\ atomic cloud.
}

By Ana Asenjo-Garcia

W hat goes up must come down, and for an atom in an excited state, "coming down" means decaying by spontaneously emitting a photon. This emission, which is triggered by vacuum fluctuations, constitutes an unavoidable source of dissipation and therefore poses a concern for quantum applications. But the moment of decay can be staved off through collective effects: when many atoms are separated by a distance smaller than the emission
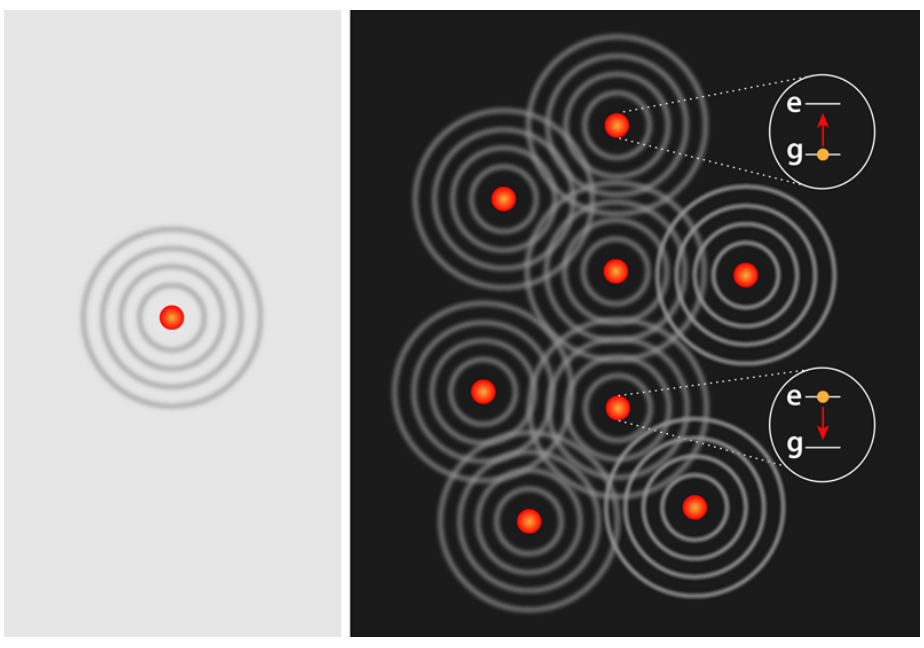

Figure 1: (Left) An atom in an excited state eventually-unavoidably-decays to its ground state, releasing a photon. (Right) In a collection of excited atoms separated by less than the transition wavelength, destructive interference in their photon emission prevents their collective decay from the excited state $(e)$ to the ground state $(g)$.

Credit: APS/Alan Stonebraker wavelength, they may become "dark," cooperatively preventing each other from decaying to the ground state. This phenomenon, called subradiance, has been previously observed in dilute atomic ensembles [1]. Now, Giovanni Ferioli and colleagues at the Institute of Optics in Palaiseau, France, have generated subradiant states in a dense atomic ensemble, in which a significant fraction of the atoms occupy a volume smaller than the emission wavelength [2]. The researchers also demonstrate that they can turn off subradiance with a laser. Such an ability to control the collective optical response of atoms in real time has implications ranging from improving the fidelity of quantum information protocols to studying out-of-equilibrium many-body physics in open quantum systems.

Subradiance is an emergent phenomenon that arises as a result of photon-mediated dipole-dipole interactions between atoms, and it is the counterpart to the more famous superradiance introduced by Robert Dicke in 1954 [3]. Photons emitted by atoms in a superradiant state interfere constructively, enhancing the overall emission (and, therefore, the decay rate). But in a subradiant state, the interference is destructive, and emission by the atomic ensemble is suppressed. Recent theoretical work has suggested the possibility of harnessing such dark states to realize more efficient protocols for photon storage and retrieval [4], metrology [5], and nonlinear quantum optics [6]. So far, the majority of research has focused on ordered arrays, with small lattice constants, which are necessary to produce a collective atomic response to light. But despite impressive developments, such as the realization of a subradiant, two-dimensional atomic mirror [7], achieving these 
small interparticle distances in ordered arrays remains a challenge.

Ferioli and colleagues are able to achive small interparticle distances by working with a very dense, disordered atomic cloud. They use a laser pulse to excite a cloud of rubidium atoms in an optical-tweezer trap, and then they record the intensity of the light radiated by the cloud. The laser mostly couples to superradiant states, and initially, the atoms decay rapidly. Subradiant states are very weakly excited, but because of their long lifetimes, they outlive superradiant states and therefore dominate emission at late times. Moreover, a fraction of the superradiant states also leaks into the subradiant states. These two processes contribute to the emergence of a long emission tail, during which the cloud radiates photons at a slow rate. The researchers investigate clouds of different geometries and demonstrate that the evolution of this subradiant emission is determined solely by the number of atoms in the cloud: as the atom number rises, the lifetime (at late times) increases. This atom-number dependence, which wouldn't be observed in a dilute system, is an important confirmation of theoretical predictions for this dense regime.

The team also explores "many-body" subradiant states in which not just one but multiple excitations are shared among the atoms. They do so by increasing the intensity of the incoming light, producing many superradiant excitations that stochastically "cascade down" toward the ground state by photon emission. This cascade may proceed in a superradiant fashion, where photons are emitted in rapid succession, but it may get "stuck" if the system reaches a dark state from which it takes a long time to decay. These dark states may be made of multiple excitations, but they still cannot radiate, because of (many-body) destructive interference. By studying the still-mysterious structure of these many-body dark states, the experiment provides the first confirmation of the theoretical prediction [4] that they are "built" from superpositions of single-excitation states. This prediction was made for ordered arrays, but the results of Ferioli and colleagues point to its validity for disordered clouds as well, although further research is needed in that direction.

As interesting as these results are, dark states become more useful if the subradiant excitations can be released at will. The researchers achieve this control by applying a position-dependent detuning of the transition, which is obtained via an ac Stark shift induced by the optical tweezer trap. This detuning disrupts the atomic resonance and brings the atoms out of their dark state, which results in a sudden burst of radiation.

So, what is next? Multiple adventures await-for experimentalists and theorists alike. Major experimental milestones would be the excitation of a single subradiant state with $100 \%$ efficiency and the selective preparation of states with specific lifetimes and spatial profiles. The method used by Ferioli and colleagues, and previously by other groups [1], reaches subradiant states by "waiting" for the system to decay into them. This approach implies an efficiency loss, as most of the energy (and information) has been released by the time the system attains the target state. Conquering this challenge might not be possible in disordered ensembles but might be more easily achieved in ordered arrays, which are attracting a lot of attention as efficient light-matter interfaces.

There are also many issues that have to be theoretically understood. Going beyond Dicke's pioneering work and understanding superradiance, subradiance and correlated decay in extended systems has been an open problem for decades. With a few exceptions $[5,8]$, most theoretical studies have focused on systems in which only a few of the atoms are excited, which makes the problem more tractable. Pushing this frontier will require a new framework to deal with the growth in exponential complexity, but the potential rewards are worthy: Understanding this out-of-equilibrium dynamics could lead to the deterministic preparation of highly entangled states with long lifetimes, which could be useful for metrology or computation. Moreover, correlated decay imprints correlations in the emitted photons, which may give rise to nonclassical states of light and may help in characterizing (and potentially controlling) this many-body system.

Ana Asenjo-Garcia: Department of Physics, Columbia University, New York, NY, USA

\section{REFERENCES}

1. W. Guerin et al., "Subradiance in a large cloud of cold atoms," Phys. Rev. Lett. 116, 083601 (2016).

2. G. Ferioli et al., "Storage and release of subradiant excitations in a dense atomic cloud," Phys. Rev. X 11, 021031 (2021). 
3. R. H. Dicke, "Coherence in spontaneous radiation processes," Phys. Rev. 93, 99 (1954).

4. A. Asenjo-Garcia et al., "Exponential improvement in photon storage fidelities using subradiance and "selective radiance" in atomic arrays," Phys. Rev. X 7, 031024 (2017).

5. L. Henriet et al., "Critical open-system dynamics in a one-dimensional optical-lattice clock," Phys. Rev. A 99, 023802
(2019).

6. R. Bekenstein et al., "Quantum metasurfaces with atom arrays," Nat. Phys. 16, 676 (2020).

7. J. Rui et al., "A subradiant optical mirror formed by a single structured atomic layer," Nature 583, 369 (2020).

8. S. J. Masson et al., "Many-body signatures of collective decay in atomic chains," Phys. Rev. Lett. 125, 263601 (2020). 